\title{
Shocked gas layers surrounding the WR nebula NGC 2359
}

\author{
J. R. Rizzo ${ }^{1,2}$, J. Martín-Pintado ${ }^{3}$, and J.-F. Desmurs ${ }^{2}$ \\ ${ }^{1}$ Departamento de Física, Universidad Europea de Madrid, Urb. El Bosque, Tajo s/n, 28670 Villaviciosa de Odón, Spain \\ 2 Observatorio Astronómico Nacional, Aptdo. Correos 1143, 28800 Alcalá de Henares, Spain \\ 3 Departamento de Astrofísica Molecular e Infrarroja, Instituto de Estructura de la Materia, CSIC, Serrano 121, \\ 28006 Madrid, Spain
}

Received 11 June 2003 / Accepted 22 August 2003

\begin{abstract}
NGC 2359 is a Wolf-Rayet (W-R) nebula partially bound by a rather dense and warm molecular cloud. We present the results derived from $\mathrm{CO}$ and ${ }^{13} \mathrm{CO}$ fully sampled maps of the molecular material with angular resolutions up to $12^{\prime \prime}$. We have detected three different velocity components, and determined their spatial distribution and physical properties. The kinematics, morphology, mass and density are clearly stratified with respect to the W-R star. These features allow us to learn about the recent evolutionary history of HD 56925, because the multiple layers could be associated to several energetic events which have acted upon the surrounding circumstellar medium. Hence, a careful study of the different shockfronts contain clues in determining the present and past interaction of this evolved massive star with its surroundings. From the analysis of the mass-loss history in massive stars like HD 56925, we suggest that the multiple layers of shocked molecular gas are likely to be produced during the earlier LBV phase and/or the actual W-R stage of HD 56925.
\end{abstract}

Key words. stars: individual: HD 56925 - stars: Wolf-Rayet - stars: winds, outflows - ISM: bubbles

- ISM: individual objects: NGC 2359 - ISM: kinematics and dynamics

\section{Introduction}

NGC 2359 is one of the archetypal wind-driven WolfRayet (W-R) nebula (Chu et al. 1983). It is an optical nebula excited by the WN4 star HD $56925\left((\mathrm{RA}, \mathrm{Dec})_{2000}=\right.$ $\left.\left(07^{\mathrm{h}} 18^{\mathrm{m}} 29^{\mathrm{s}} .13,-13^{\circ} 13^{\prime} 01^{\prime \prime} .5\right)\right)$, which is catalogued as WR7 by van der Hucht (2001). NGC 2359 has an almost spherical morphology, and contains some $\mathrm{N}$-enriched filaments inside (Esteban et al. 1990). The southern part of the nebula - which contains most of the associated molecular gas - has been observed in the recent years.

St-Louis et al. (1998) have detected the $\mathrm{H}_{2}$ 1-0 S(1) emission in coincidence with $\mathrm{H} \alpha$, but they could not disentangle the dominant excitation mechanism of the molecular hydrogen. Carbon monoxide was firstly detected by Schneps et al. (1981), and subsequently mapped in the $J=1 \rightarrow 0$ and $2 \rightarrow 1$ lines by Rizzo et al. (2001a, Paper I) and by Cappa et al. (2001). While Cappa et al. focussed in determining the total neutral mass and in the energetics, Paper I has concentrated on the interaction processes between the nebula and the surrounding molecular gas.

In Paper I, it was shown that the ambient molecular cloud, observed at a velocity in the Local Standard of Rest $\left(V_{\mathrm{LSR}}\right)$ of $67 \mathrm{~km} \mathrm{~s}^{-1}$, was accelerated to $V_{\mathrm{LSR}}=53-54 \mathrm{~km} \mathrm{~s}^{-1}$ by the stellar wind from HD 56925. It was also established the

Send offprint requests to: J. R. Rizzo,

e-mail: jricardo.rizzo@fis.cie.uem.es presence of a rather hot region just coincident with the $\mathrm{H}_{2}$ vibrationally excited emission surrounding the southern optical nebula, as well as a high-opacity zone adjacent to the cold quiescent cloud. Observations of ${ }^{13} \mathrm{CO}$ have revealed another striking feature: in the region of higher intensity, the ${ }^{13} \mathrm{CO} J=$ $1 \rightarrow 0$ line peaks at $54-55 \mathrm{~km} \mathrm{~s}^{-1}, 1-2 \mathrm{~km} \mathrm{~s}^{-1}$ higher than the $\mathrm{CO} J=1 \rightarrow 0$ and the $J=2 \rightarrow 1$ lines. It is worth mentioning that, at the same position, the metastable $(1,1)$ and $(2,2)$ lines of ammonia $\left(\mathrm{NH}_{3}\right)$ were detected for the first time in a W-R nebula (Rizzo et al. 2001b). From the $\mathrm{NH}_{3}$ data, a rotational temperature of $30 \mathrm{~K}$ and a relatively high abundance of $10^{-8}$ were determined.

These observational findings indicate the nature of the interaction with the ambient material during different evolutive stages from the hot star. The molecular cloud has been exposed to the UV radiation from the hot star and to the shocks produced by the stellar winds from the W-R star and the mass ejections from previous stages. According to the hydrodynamical models of García-Segura et al. (1996a,b), several shocks are expected at a few pc from a massive evolved star, as a consequence of the time-dependent stellar winds of the postmain sequence evolution, mainly the red supergiant (RSG) and the luminous blue variable (LBV) phases. Hence, the molecular cloud surrounding NGC 2359 provides us with an excellent laboratory to study the interaction between the massive stars and their environment. W-R stars will disturb the circumstellar medium (CSM), generating an ionized nebula 
surrounded by a shocked region. In particular, the $\mathrm{NH}_{3}$ would be a good molecule to disentangle the effect of the radiative field and the shock in the environment of W-R stars. The relatively high abundance of $\mathrm{NH}_{3}$ strongly suggests a shock chemistry in the outer part of the cloud, since it would be released from the dust grain mantles by non-dissociative shocks (Flower \& Pineau des Forêts 1994; Rizzo et al. 2001b).

A detailed knowledge of the spatial and kinematical distributions of the $\mathrm{CO}$ isotopes would allow us to know with more precision the physical conditions and the kinematical features in this scenario, together with an approach to the knowledge of the dominant mechanisms of excitation (UV fields or shocks). In order to satisfy these goals, we combine in this paper high angular resolution $\mathrm{CO} J=1 \rightarrow 0, \mathrm{CO} J=2 \rightarrow 1$ and ${ }^{13} \mathrm{CO} J=1 \rightarrow 0$ maps with high sensitivity spectra of $\mathrm{CO},{ }^{13} \mathrm{CO}$ and $\mathrm{C}^{18} \mathrm{O}$ taken at selected positions. This observational study reveals the presence of hot dense regions, warm regions and, more conclusively, the stratified distribution of different kinematical components, associated to several shockfronts which have been acting in the recent past of HD 56925.

\section{Observations}

We have used the IRAM 30-m radiotelescope at Pico Veleta (Spain), during two observing runs in June and August, 2001. We have performed two different observing modes, namely on-the-fly maps and single position observations.

The on-the-fly maps were obtained for the $\mathrm{CO}$ and ${ }^{13} \mathrm{CO} J=$ $1 \rightarrow 0$, and the CO $J=2 \rightarrow 1$ lines simultaneously. We have mapped a $240^{\prime \prime} \times 120^{\prime \prime}$ area toward the southern part of NGC 2359. The scanning directions were orthogonal in the equatorial system, e.g., at constant right ascension and constant declination. Row spacing between scans were $6^{\prime \prime}$, and therefore all spectral lines maps are fully sampled. Individual maps were later combined using the GILDAS package. One receiver was tuned for each $J=1 \rightarrow 0$ line, while the CO $J=2 \rightarrow 1$ line was observed simultaneously by two different receivers. A 256-channels filter bank with $100 \mathrm{kHz}$ of channel resolution and a 2048-channel autocorrelator were used as backends, providing velocity resolutions between 0.1 and $0.3 \mathrm{~km} \mathrm{~s}^{-1}$. Typical rms was $0.3 \mathrm{~K}$ for all the observed lines. All the temperatures throughout this paper are referred to an atmosphere corrected scale, e.g., $T_{\mathrm{A}}^{*}$.

The single position observations were performed along a strip of particular interest in position-switching mode. We observed the $J=1 \rightarrow 0$ and $J=2 \rightarrow 1$ lines of $\mathrm{CO}$ and ${ }^{13} \mathrm{CO}$ simultaneously, with integration time between 15 and 40 minutes. As a byproduct, the $\mathrm{C}^{18} \mathrm{O} J=1 \rightarrow 0$ line was also observed with the same receiver than the ${ }^{13} \mathrm{CO} J=1 \rightarrow 0$ line. We used the same backend as for the on-the-fly maps, and hence we had identical velocity resolutions. The achieved rms was better than $50 \mathrm{mK}$ in all cases.

Calibration was performed during the observations and later checked up on standard sources (Mauersberger et al. 1989). The HPBW was $22^{\prime \prime}$ for the CO $J=1 \rightarrow 0$ line, $11^{\prime \prime}$ for the $\mathrm{CO} J=2 \rightarrow 1$ line, $24^{\prime \prime}$ for the ${ }^{13} \mathrm{CO} J=1 \rightarrow 0$ line and $12^{\prime \prime}$ for the ${ }^{13} \mathrm{CO} J=2 \rightarrow 1$ line.

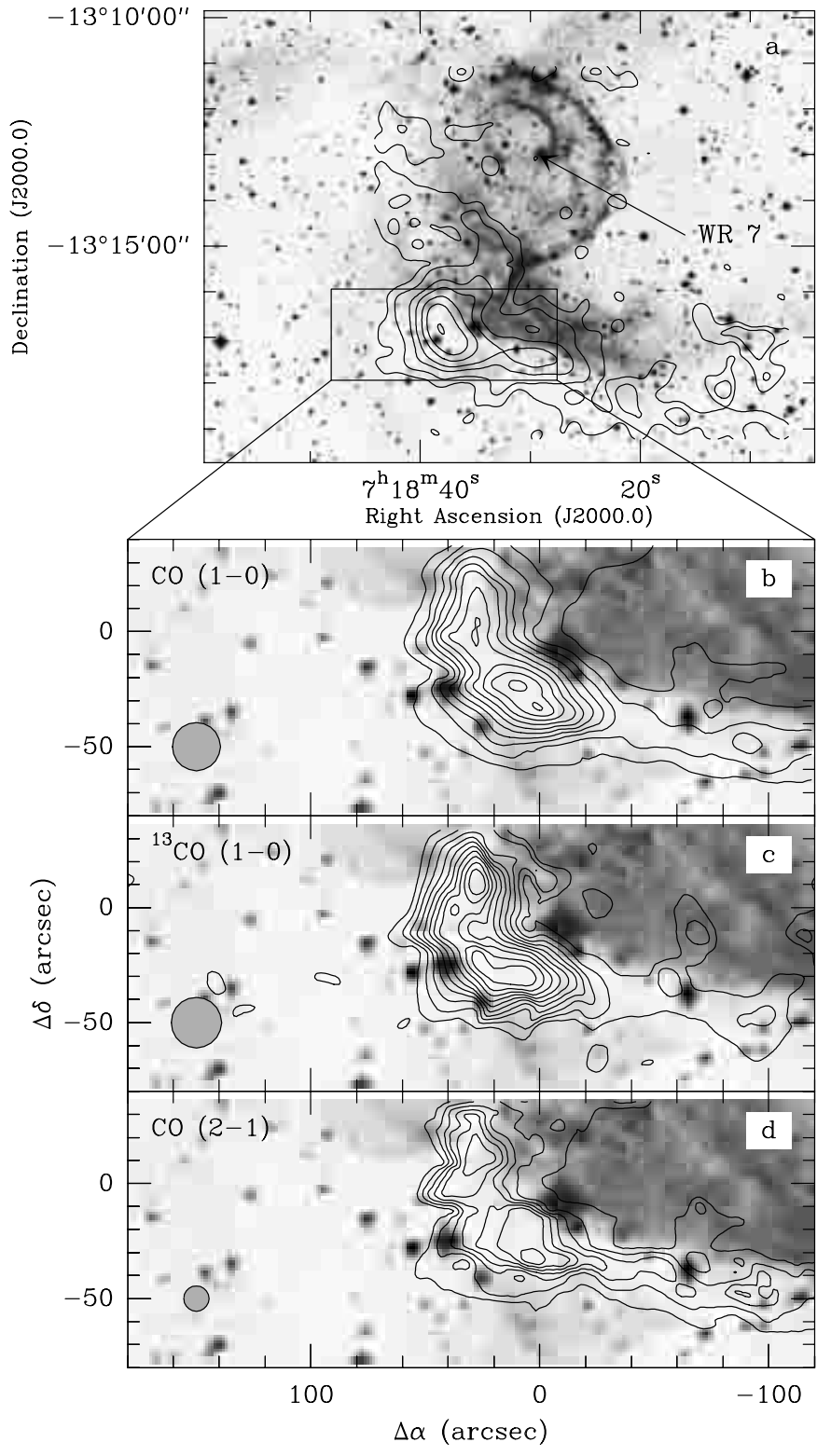

Fig. 1. $\mathrm{CO}$ and ${ }^{13} \mathrm{CO}$ integrated emission from 50 to $56 \mathrm{~km} \mathrm{~s}^{-1}$ toward NGC 2359. a) $J$-band picture of the optical nebula, overlaid with the CO $J=2 \rightarrow 1$ emission from Rizzo et al. (2001a). The rectangle indicates the region mapped in this paper with higher angular resolution. b), c) and d) Molecular transitions indicated at the top left of each map, with the beams plotted in the bottom left corner. Angular offsets in Figs. b), c) and d) are referred to (RA, Dec) ${ }_{2000}=$ $\left(07^{\mathrm{h}} 18^{\mathrm{m}} 35^{\mathrm{s}} .7,-13^{\circ} 16^{\prime} 37^{\prime \prime} \cdot 0\right)$. First level and spacing in these figures are $4.4,0.8$ and $4 \mathrm{~K} \mathrm{~km} \mathrm{~s}^{-1}$, respectively.

\section{Results}

\subsection{On-the-fly maps}

The southern part of the nebula was mapped in the $\mathrm{CO} J=$ $1 \rightarrow 0, J=2 \rightarrow 1$ and ${ }^{13} \mathrm{CO} J=1 \rightarrow 0$ lines simultaneously. In Paper I, it has been shown that this region contains the most intense and interesting $\mathrm{CO}$ features, between 52 and $67 \mathrm{~km} \mathrm{~s}^{-1}$. The maps in Fig. 1 show the integrated emission of these lines from 50 to $56 \mathrm{~km} \mathrm{~s}^{-1}$. Figure 1a shows the whole CO $J=2 \rightarrow 1$ emission from Paper I, superimpossed to an optical image of 


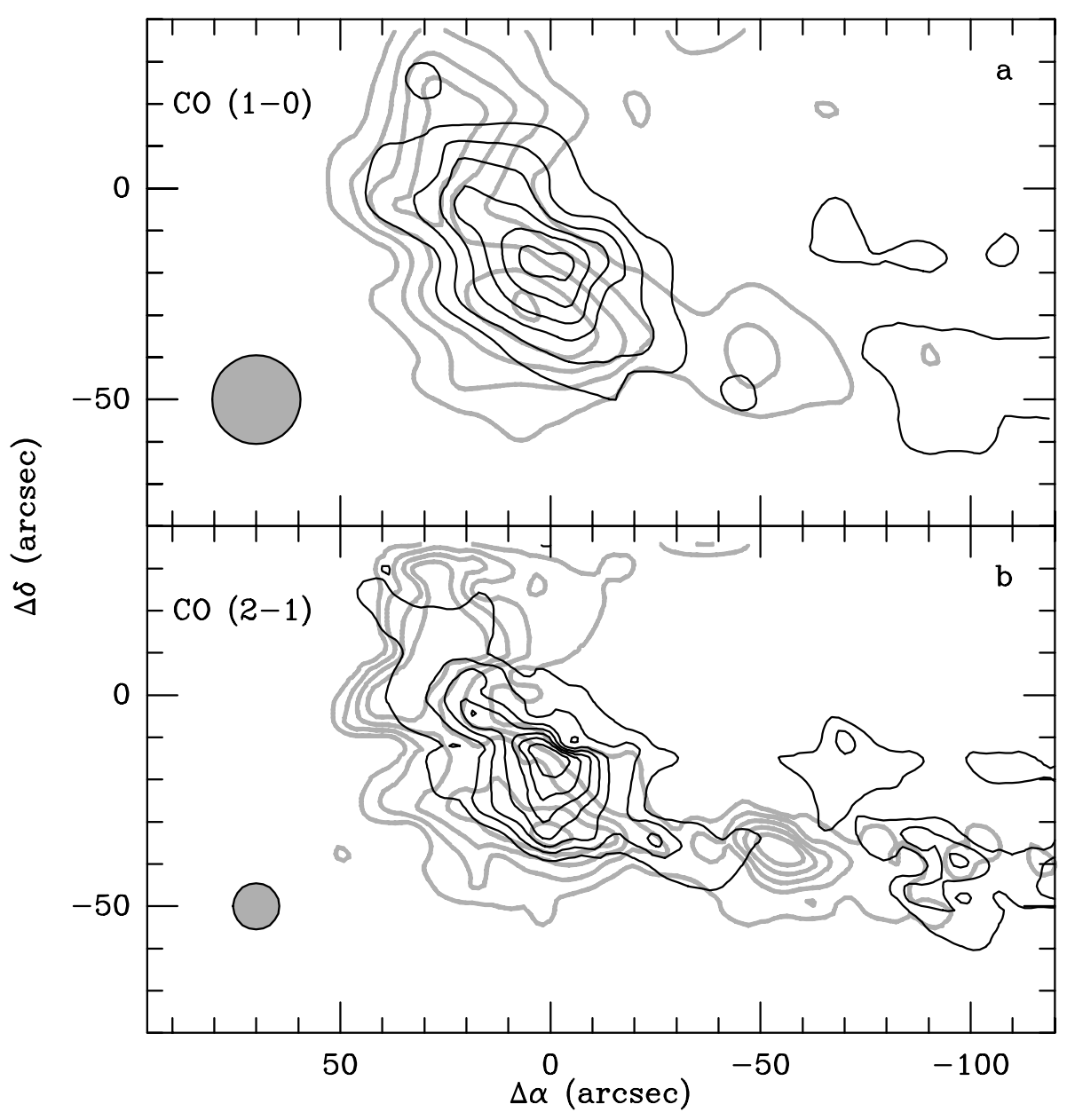

Fig. 2. Comparison of the $\mathrm{CO}$ emission in two velocity channels. a) $\mathrm{CO} J=1 \rightarrow 0$ brightness temperature at $55.2 \mathrm{~km} \mathrm{~s}^{-1}$ (grey contours), overlaid with the emission at $50.1 \mathrm{~km} \mathrm{~s}^{-1}$ (black contours). Lower contour and step are $0.3 \mathrm{~K}$ (grey) and $0.16 \mathrm{~K}$ (black). b) The same as a) for the $\mathrm{CO} J=2 \rightarrow 1$ brightness temperature. Lower contour and step are $0.15 \mathrm{~K}$ (grey) and $0.8 \mathrm{~K}$ (black). We note in both maps a clear shift in the emission, separated by $\sim 20^{\prime \prime}$.

the nebula. The same figure also indicates the region mapped in this work. Figures $1 \mathrm{~b}-1 \mathrm{~d}$ show the results of these data in the three spectral lines already mentioned. We can see that the molecular cloud perfectly matchs the southern border of the optical nebula. The $\mathrm{CO}$ and ${ }^{13} \mathrm{CO}$ emission are confined to layers which are less than $70^{\prime \prime}$ wide.

The overall emission maps depicted in Fig. 1 do not show significant differences among the observed spectral lines. However, a number of new features appear when the emission is analysed in smaller velocity bins. Figure 2 shows an overlay of the CO $J=1 \rightarrow 0$ and $J=2 \rightarrow 1$ emissions in two different channel at velocities of $55.2 \mathrm{~km} \mathrm{~s}^{-1}$ (grey contours) and $50.1 \mathrm{~km} \mathrm{~s}^{-1}$ (black contours). It is clearly noted a separation of more than $20^{\prime \prime}$ between the emission at both velocities. Actually, the thinner layer located toward the inner part of the nebula, at a velocity of $\sim 50 \mathrm{~km} \mathrm{~s}^{-1}$, is adjacent to the more extended emission. The width of this layer is of about $15-20^{\prime \prime}$, as we can see in the $\mathrm{CO} J=2 \rightarrow 1$ map.

To better understand the velocity structure of the $\mathrm{CO}$ emission we have analyzed the data along several position-velocity strips. The most illustrative one is shown in Fig. 3. This strip crosses both the large and the thin molecular layers. It is clearly shown a sudden line broadening just at the position of the thin layer, around $50 \mathrm{~km} \mathrm{~s}^{-1}$. This behaviour clearly demonstrates that the feature noted in Fig. 2 is not merely due to a velocity gradient of a single component, but to the appearing of a second component only present at the interface between the molecular cloud and the optical nebula.

\subsection{Individual positions}

In order to have a complete picture of this striking feature, we performed high-sensitivity, individual observations at the positions shown as squares in Fig. 3a. These positions, labelled with letters from A to E, are spaced by $\sim 20^{\prime \prime}$. Observed spectral lines at these locations are the $\mathrm{CO} J=1 \rightarrow 0, \mathrm{CO} J=$ $2 \rightarrow 1,{ }^{13} \mathrm{CO} J=1 \rightarrow 0{ }^{13} \mathrm{CO} J=2 \rightarrow 1$, and $\mathrm{C}^{18} \mathrm{O} J=$ $1 \rightarrow 0$ lines. The ${ }^{13} \mathrm{CO} J=2 \rightarrow 1$ and $\mathrm{C}^{18} \mathrm{O} J=1 \rightarrow 0$ lines were detected for the first time in a W-R environment.

The resulting spectra are shown in Fig. 4. For all the observed lines, the peak velocity remains roughly constant from positions $\mathrm{A}$ to $\mathrm{C}$, and the linewidth increases. Both $\mathrm{CO}$ lines at position $\mathrm{C}$ are stronger than at position $\mathrm{B}$, while in both ${ }^{13} \mathrm{CO}$ lines we note exactly the contrary. Toward 

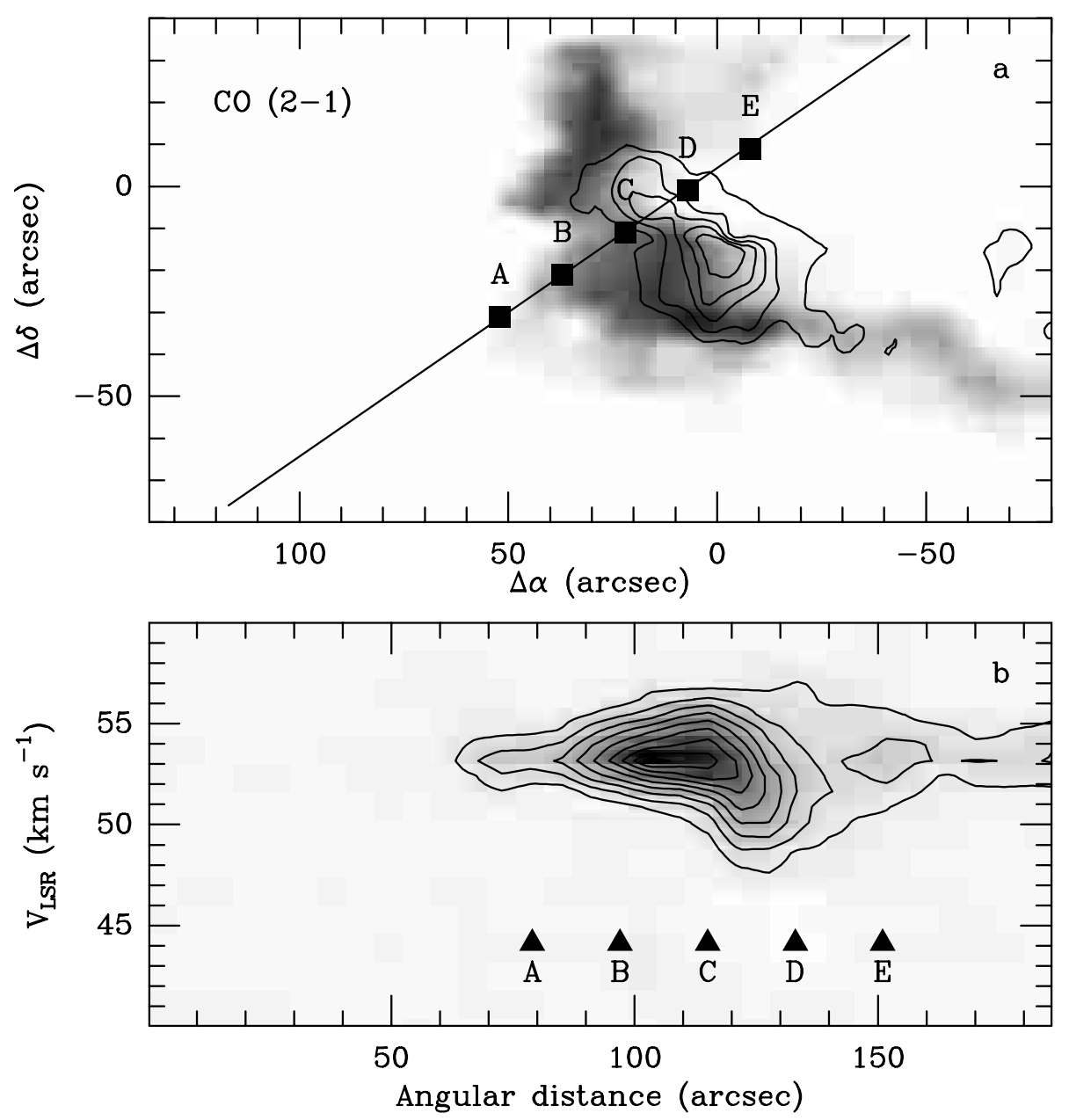

Fig. 3. A position-velocity strip in the CO $J=2 \rightarrow 1$ emission. a) CO $J=2 \rightarrow 1$ emission map integrated between 52 and $55 \mathrm{~km} \mathrm{~s}^{-1}$ (on greyscale, from 2 to $22 \mathrm{~K} \mathrm{~km} \mathrm{~s}^{-1}$ ) and between 48 and $51 \mathrm{~km} \mathrm{~s}^{-1}$ (contours, 1 to 5 times $0.2 \mathrm{~K} \mathrm{~km} \mathrm{~s}^{-1}$ ). The straight line indicates the location of the strip. The filled squares along the line show the individual positions observed with higher sensitivity (see Sect. 3.2 and Fig. 4). The three kinematical components described in Sect. 4 are distinguished, since the greyscale represents the low velocity component, the contours sketches the intermediate velocity component and the high velocity component is detected just in position E. b) Position-velocity strip in the velocity range of interest. Angular distance is measured from southeast to northwest. The bottom small triangles indicate the approximate location of the individual positions marked by squares in a). Contours are 1 to 10 times $0.6 \mathrm{~K}$. In general, we do not see a velocity variation greater than $1 \mathrm{~km} \mathrm{~s}^{-1}$ along the slice. However, a striking line broadening toward the inner part of the nebula appears, in close coincidence with the thin layer at $48-50 \mathrm{~km} \mathrm{~s}^{-1}$ depicted in Fig. 2.

position D all the line intensities are roughly reduced to one half and a strong assymetry and line broadening appear. There is significant emission at velocities as low as $47 \mathrm{~km} \mathrm{~s}^{-1}$. The broadening shown in the position-velocity strip (Fig. 3b) is coincident with this position. $\mathrm{C}^{18} \mathrm{O}$ is only detected in positions $\mathrm{B}$ and $\mathrm{C}$, corresponding to the highest emission at the other isotopes.

Position E, although weak, shows a large CO $J=2 \rightarrow 1$ broadening to velocities as low as $42 \mathrm{~km} \mathrm{~s}^{-1}$. This implies a total velocity interval emission of at least $14 \mathrm{~km} \mathrm{~s}^{-1}$. It is worth mentioning that this very broad emission at $V_{\mathrm{LSR}}<50 \mathrm{~km} \mathrm{~s}^{-1}$ is not detected in the $\mathrm{CO} J=1 \rightarrow 0$ line. As mentioned above, we see in Fig. $3 \mathrm{~b}$ a notable line broadening between positions $\mathrm{C}$ and $\mathrm{D}$, where the lower levels are seen at 47 and $57 \mathrm{~km} \mathrm{~s}^{-1}$. Surprisingly, the high sensitivity individual positions reveals a third velocity component at position $\mathrm{E}$, which could not be detected using the on-the-fly maps because of the sensitivity.
The main observational findings are summarized in Table 1. For each position, this table shows the parameters of the five observed transitions, obtained from a Gaussian fit to a single component: antenna temperature of the maximum $\left(T_{\mathrm{pk}}\right)$, LSR peak velocity $\left(V_{\mathrm{LSR}}\right)$ and full width at half maximum $(\Delta \mathrm{V})$. The single component assumption is obviously not valid in some cases due to the strong assymetries of the line profiles, and hence is only provided for comparison.

\section{Physical parameters}

\subsection{Velocity components}

The observational features shown in Sect. 3 (in particular Figs. 3 and 4, and Table 1) lead us to define three different velocity components. The first component corresponds to the most extended molecular gas and is the broad component, already studied in Paper I, at velocities from 52 to $57 \mathrm{~km} \mathrm{~s}^{-1}$. 

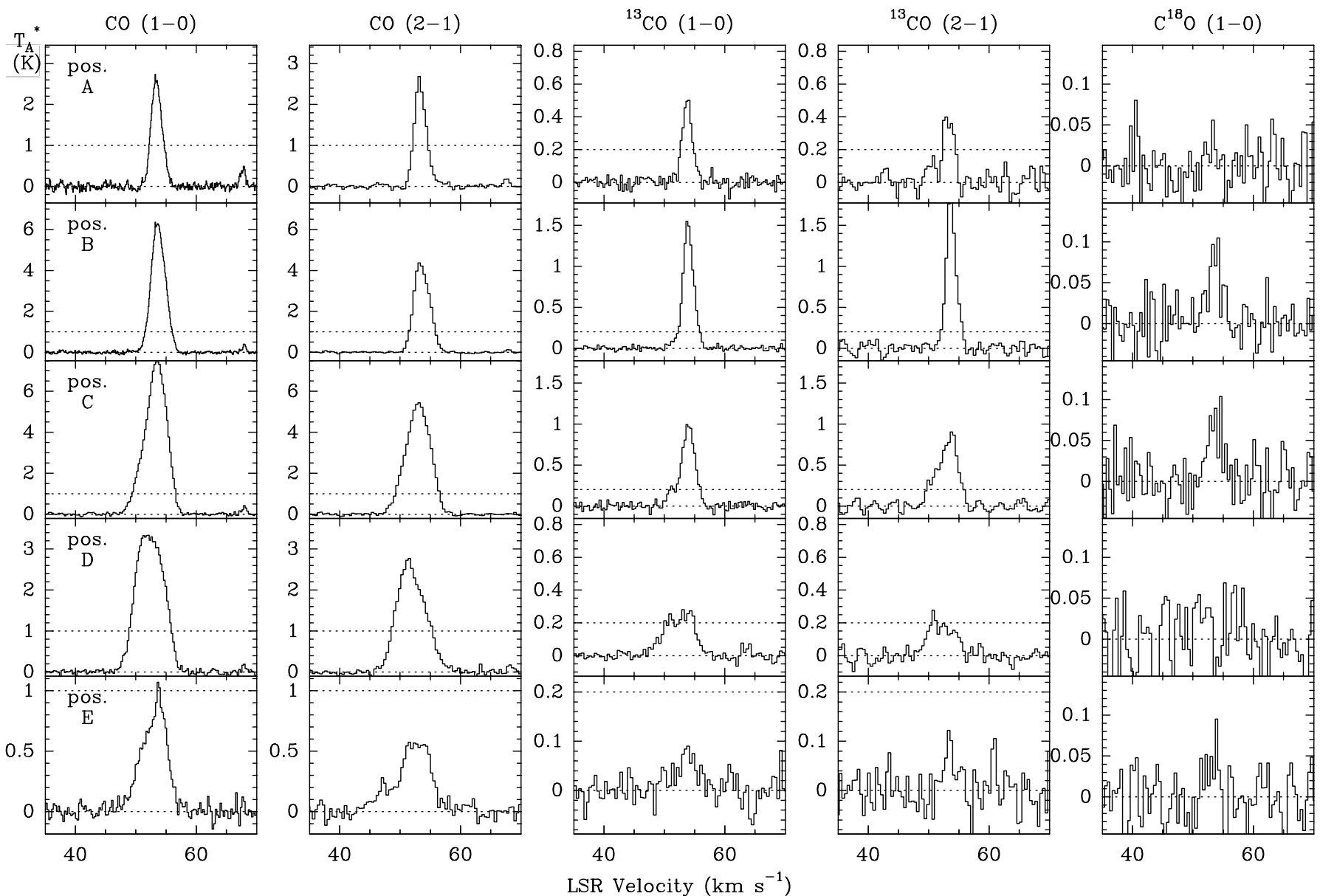

Fig. 4. High-sensitivity, individual spectra of CO isotopes along the strip shown in Fig. 3. Every column corresponds to the spectral line written at the top, while every row corresponds to the selected positions. The assymetries in positions $\mathrm{C}, \mathrm{D}$ and $\mathrm{E}$ indicates the presence of different velocity components. It is striking the very broad emission of the $\mathrm{CO} J=2 \rightarrow 1$ line in position E. ${ }^{13} \mathrm{CO} J=2 \rightarrow 1$ and $\mathrm{C}^{18} \mathrm{O} J=1 \rightarrow 0$ are detected for the first time in a W-R nebula. Temperature scale is $T_{\mathrm{A}}^{*}(\mathrm{~K})$. Since different scales are used, auxiliary lines at $1 \mathrm{~K}$ (for both $\mathrm{CO}$ lines) and $0.2 \mathrm{~K}$ (for the ${ }^{13} \mathrm{CO}$ lines) were also plotted.

Table 1. Gaussian fits to the observed CO isotopic spectral lines.

\begin{tabular}{|c|c|c|c|c|c|c|c|c|c|c|c|c|c|c|c|}
\hline \multirow[t]{3}{*}{ Pos. } & \multicolumn{3}{|c|}{$\overline{C O O J=1 \rightarrow 0}$} & \multicolumn{3}{|c|}{$\overline{\mathrm{CO} J=2 \rightarrow 1}$} & \multicolumn{3}{|c|}{$\overline{{ }^{13} \mathrm{CO} J=1 \rightarrow 0}$} & \multicolumn{3}{|c|}{${ }^{13} \mathrm{CO} J=2 \rightarrow 1$} & \multicolumn{3}{|c|}{$\mathrm{C}^{18} \mathrm{O} J=1 \rightarrow 0$} \\
\hline & $T_{\mathrm{pk}}$ & $V_{\mathrm{LSR}}$ & $\Delta V$ & $T_{\mathrm{pk}}$ & $V_{\mathrm{LSR}}$ & $\Delta V$ & $T_{\mathrm{pk}}$ & $V_{\mathrm{LSR}}$ & $\Delta V$ & $T_{\mathrm{pk}}$ & $V_{\mathrm{LSR}}$ & $\Delta V$ & $T_{\mathrm{pk}}$ & $V_{\mathrm{LSR}}$ & $\Delta V$ \\
\hline & $\mathrm{K}$ & \multicolumn{2}{|c|}{$\mathrm{km} \mathrm{s}^{-1}$} & $\mathrm{~K}$ & \multicolumn{2}{|c|}{$\mathrm{km} \mathrm{s}^{-1}$} & $\mathrm{~K}$ & \multicolumn{2}{|c|}{$\mathrm{km} \mathrm{s}^{-1}$} & $\mathrm{~K}$ & \multicolumn{2}{|c|}{$\mathrm{km} \mathrm{s}^{-1}$} & $\mathrm{~K}$ & \multicolumn{2}{|c|}{$\mathrm{km} \mathrm{s}^{-1}$} \\
\hline A & 2.6 & 53.4 & 2.1 & 2.6 & 53.3 & 2.3 & 0.5 & 53.8 & 1.9 & 0.4 & 53.2 & 2.2 & & $04 \mathrm{~K}$ & $\overline{\mathrm{s}^{-1 a}}$ \\
\hline B & 6.3 & 53.7 & 2.8 & 4.4 & 53.6 & 3.1 & 1.5 & 54.0 & 2.1 & 1.8 & 53.7 & 1.9 & 0.08 & 53.6 & 2.6 \\
\hline $\mathrm{C}$ & 7.4 & 53.3 & 4.2 & 5.4 & 53.0 & 4.7 & 1.0 & 53.9 & 2.7 & 0.8 & 53.2 & 4.0 & 0.08 & 53.7 & 2.8 \\
\hline D & 3.6 & 52.3 & 5.3 & 2.6 & 51.8 & 5.5 & 0.3 & 52.5 & 5.8 & 0.2 & 52.0 & 5.2 & & $09 \mathrm{~K} 1$ & $\mathrm{~s}^{-1 a}$ \\
\hline $\mathrm{E}$ & 0.9 & 53.2 & 4.8 & 0.6 & 53.0 & 14.0 & 0.1 & 53.7 & 4.3 & 0.1 & 53.4 & 1.5 & & $05 \mathrm{~K} 1$ & $\mathrm{~s}^{-1 a}$ \\
\hline
\end{tabular}

${ }^{a}$ Upper limit of the integrated line intensity.

In the following, we shall refer to this component as the "low velocity component" $(l v c)$. The higher spatial resolution of our maps allow us to disclose the presence of a second, less extended velocity component, seen as a thin layer $\left(\sim 20^{\prime \prime}\right.$ width $)$ in Fig. 2, in the velocity range $48-52 \mathrm{~km} \mathrm{~s}^{-1}$. We shall refer to this component as the "intermediate velocity component" (ivc). Finally, the high sensitivity of the individual spectra revealed the third component, hereafter referred to as the "high velocity component" $(h v c)$, as a very compact one, clearly detected only in the $\mathrm{CO} J=2 \rightarrow 1$ line toward position E (Fig. 4), at velocities lower than $48 \mathrm{~km} \mathrm{~s}^{-1}$.

The extension in the sky corresponding to each component may be roughly inferred from Fig. 3a: the $l v c$ is sketched in the greyscale, the $i v c$ is plotted in contours, and the $h v c$ is only detected in position E. The $l v c$ corresponds to most of the gas detected in Paper I. It is the most extended component, and bounds the whole optical nebula by its southern and eastern borders. The ivc is at the interface between NGC 2359 and the 


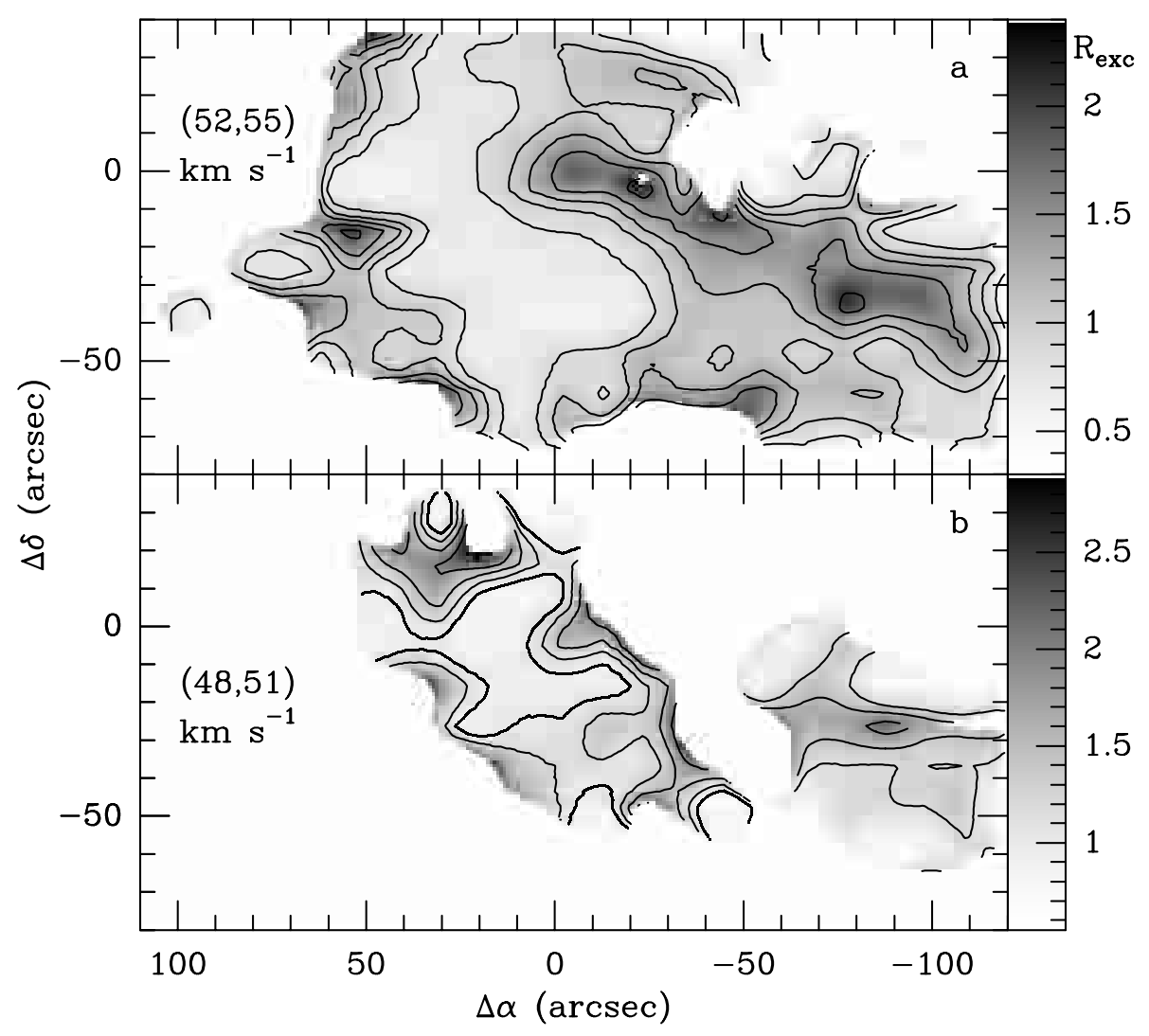

Fig. 5. CO $J=2 \rightarrow 1$ to $J=1 \rightarrow 0$ line ratio $\left(R_{\text {exc }}\right)$ in the southern part of NGC 2359 . The velocity ranges are indicated at the left of each figure. a) "low velocity component". b) "intermediate velocity component". In general, $R_{\text {exc }}$ does not show no large changes in both velocity intervals, except toward the inner nebula, in panel a), where $R_{\mathrm{exc}}$ is at least a factor of six larger than the mean value.

most intense emission of $l v c$. It seems to form a narrow layer between the $l v c$ and the optical nebula. Finally, the position where the $h v c$ was detected is projected onto the ionized gas and presumably has a compact morphology.

The stratified location of the three components with respect to the optical nebula NGC 2359 and the quiescent molecular gas at $67 \mathrm{~km} \mathrm{~s}^{-1}$, might be explained by succesive events associated to the evolution of a massive star. Therefore, different ages are expected for each component. The $l v c$ is the most extended, uniform and massive one, its velocity is the closest to the quiescent gas and hence is thought as the oldest one. The $i v c$, however, is less extended than the $l v c$ and closer to the W-R star. These kinematical and morphological features might be explained by an event faster and younger than that which formed the $l v c$. So far, the $i v c$ may be the result of the encounter of two winds, in a manner suggested by García-Segura et al. (1996a,b). Finally, the hvc may be recently shocked and would be the youngest component.

\subsection{Overall features}

Our on-the-fly $\mathrm{CO}$ and ${ }^{13} \mathrm{CO}$ maps trace the distribution of the molecular gas both into and around the southern optical nebula, including the interesting molecular interface at its border. The different velocity components have probably been produced by violent energetic mechanisms usually linked to the massive star evolution. During the evolution of HD 56925, the stellar wind, eventual envelope ejections and the strong UV radiation fields could have produced shocked, ionized and photodissociated regions. The relative variations of different spectral lines should tell us about the physical conditions of the molecular gas.

We have analyzed the line ratios among the three observed spectral lines which covers the southern and eastern borders of NGC 2359. In the following, we shall refer to the line ratio of ${ }^{12} \mathrm{CO} J=1 \rightarrow 0$ to ${ }^{13} \mathrm{CO} J=1 \rightarrow 0$ as $R_{\text {isot }}$, and to the line ratio of ${ }^{12} \mathrm{CO} J=2 \rightarrow 1$ to ${ }^{12} \mathrm{CO} J=1 \rightarrow 0$ as $R_{\text {exc }}$. Both $R_{\text {isot }}$ and $R_{\text {exc }}$ were computed for different velocity ranges and for positions with line intensity greater than $3 \sigma . R_{\mathrm{exc}}$ was computed after convolving the $\mathrm{CO} J=2 \rightarrow 1$ map to the $J=$ $1 \rightarrow 0$ angular resolution. While $R_{\text {exc }}$ is a good tracer of the excitation temperature, $R_{\text {isot }}$ might trace the combined effect of isotopic variations and changes in the $\mathrm{CO}$ opacity.

Figures $5 \mathrm{a}$ and $5 \mathrm{~b}$ show the spatial distribution of $R_{\text {exc }}$ for the velocity ranges $(52,55)$ and $(48,51) \mathrm{km} \mathrm{s}^{-1}$, roughly equivalent to the $l v c$ and the $i v c$, respectively. For the $l v c$ (Fig. 5a), $R_{\text {exc }}$ does not significantly changes in the brightest zone, and it is probably greater toward the south. A mean value for $R_{\text {exc }}$ of 0.9 and 1.2 is found to the eastern and southern part of the map, respectively. We have obtained similar results for the ivc (Fig. 5b), where a mean value of 1 is found. However, the ${ }^{13} \mathrm{CO}$ emission is really striking when compared to the $\mathrm{CO} J=1 \rightarrow 0$ emission. Figure 6 sketches the distribution of $R_{\text {isot }}$ in the velocity interval $(52,55) \mathrm{km} \mathrm{s}^{-1}$ (roughly the $l v c$ ). It clearly changes sistematically from southeast to northwest, and varies in almost one order of magnitude. The sensitivity of our on-the-fly maps were not enough to 


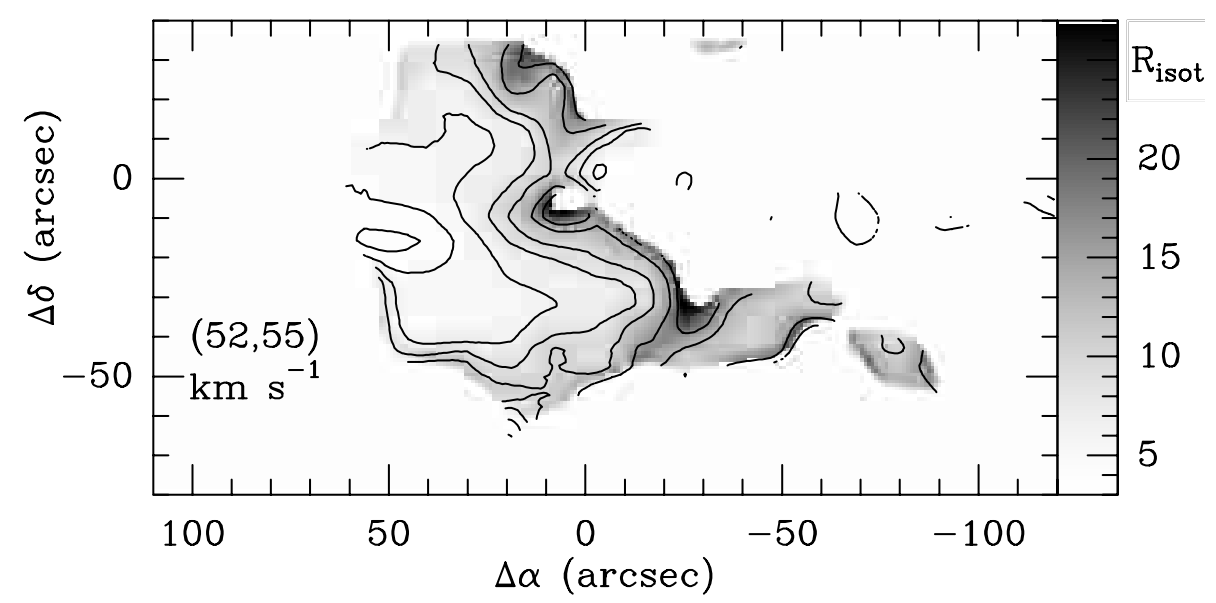

Fig. 6. CO to ${ }^{13} \mathrm{CO} J=1 \rightarrow 0$ line ratio $\left(R_{\mathrm{isot}}\right)$ in the southern part of NGC 2359 . The velocity range of integration is $(52,55) \mathrm{km} \mathrm{s}^{-1}$. A systemic change (almost by one order of magnitude) from east to west is noted all over the map. This might indicate a different distribution of $\mathrm{CO}$ and ${ }^{13} \mathrm{CO}$, variation of abundance or opacity effects in the area.

Table 2. LVG results for the CO isotopes in NGC 2359.

\begin{tabular}{llccccc}
\hline \hline Pos. & Comp. & $\begin{array}{c}n\left(\mathrm{H}_{2}\right) \\
10^{3} \mathrm{~cm}^{-3}\end{array}$ & $\begin{array}{c}N(\mathrm{CO}) \\
10^{16} \mathrm{~cm}^{-2}\end{array}$ & $\begin{array}{c}N\left({ }^{13} \mathrm{CO}\right) \\
10^{14} \mathrm{~cm}^{-2}\end{array}$ & $\begin{array}{c}N\left(\mathrm{C}^{18} \mathrm{O}\right) \\
10^{14} \mathrm{~cm}^{-2}\end{array}$ & ${ }^{13} \mathrm{CO} / \mathrm{C}^{18} \mathrm{O}$ \\
\hline $\mathrm{A}$ & $l v c$ & 3.0 & 6.1 & 10.2 & $<0.8$ & $>12.9$ \\
$\mathrm{~B}$ & $l v c$ & 4.9 & 24.4 & 40.7 & 2.2 & 18.2 \\
$\mathrm{C}$ & $l v c$ & 3.0 & 17.3 & 28.8 & 1.9 & 14.8 \\
$\mathrm{C}$ & $i v c$ & 11.5 & 3.8 & 6.3 & $<1.5$ & $>4.3$ \\
$\mathrm{D}$ & $l v c$ & 3.9 & 3.5 & 5.9 & $<1.3$ & $>4.4$ \\
$\mathrm{D}$ & $i v c$ & 3.0 & 5.3 & 8.9 & $<1.4$ & $>6.5$ \\
$\mathrm{E}$ & $l v c$ & 1.4 & 1.7 & 2.8 & $<0.8$ & $>3.5$ \\
$\mathrm{E}$ & $i v c$ & 1.7 & 0.2 & $<0.7$ & $<1.0$ & - \\
$\mathrm{E}$ & $h v c$ & $\gtrsim 100$ & 0.2 & $<2.0$ & $<1.6$ & - \\
\hline
\end{tabular}

detect the ${ }^{13} \mathrm{CO}$ emission in the velocity range corresponding to the $i v c$, and $R_{\text {isot }}$ could not be computed.

\subsection{Excitation conditions}

From the overall information shown in Figs. 5 and 6, we see a rather uniform excitation conditions over the whole region. So far, in most of the observed area, the excitation temperature does not varies significantly. However, large variations in the $\mathrm{CO}$ opacity might be inferred. If we assume a constant ${ }^{13} \mathrm{CO} / \mathrm{CO}$ isotopic abundance, the $\mathrm{CO}$ lines will be optically thick in the eastern part of the cloud. This feature explains the velocity difference between both isotopes, as noted in Paper I.

We have estimated the physical conditions for the three velocity components, by modelling the emission of the observed lines for positions A to $\mathrm{E}$, using the large velocity gradient method (LVG). The LVG method implements the Sobolev's formulation developed by Castor (1970) and later extended by Jeffery (1995), among others. We have computed the parameters assuming kinetic temperatures $\left(T_{\mathrm{K}}\right)$ from 10 to $100 \mathrm{~K}$. For all components but the $h v c$, a minimum $T_{\mathrm{K}}$ of $30 \mathrm{~K}$ is needed to match the observed lines. This lower limit for $T_{\mathrm{K}}$ is in good agreement with the results of Rizzo et al. (2001b), based on the metastable $\mathrm{NH}_{3}(1,1)$ and $(2,2)$ lines detected at position B. Furthermore, the results are not critically different for the temperature range $30-100 \mathrm{~K}$. Both CO lines result to be optically thick in most cases, whereas both ${ }^{13} \mathrm{CO}$ lines were optically thin in all cases. In consequence, we used the ${ }^{13} \mathrm{CO} 2-1 / 1-0$ line ratio (on a main-beam temperature scale) to estimate the $\mathrm{H}_{2}$ density, $n\left(\mathrm{H}_{2}\right)$. For a given $T_{\mathrm{K}}$, the column densities of ${ }^{13} \mathrm{CO}$ and $\mathrm{C}^{18} \mathrm{O}$ were determined from the derived $n\left(\mathrm{H}_{2}\right)$ and the $J=1 \rightarrow 0$ line intensity of each isotope. The CO column density was estimated by assuming a constant isotopic ratio of 60 between $\mathrm{CO}$ and ${ }^{13} \mathrm{CO}$ (Wilson \& Matteucci 1992)

The Table 2 shows the results of applying the LVG method to each component at each position, by assuming a $T_{\mathrm{K}}$ of $30 \mathrm{~K}$. By adopting this value for $T_{\mathrm{K}}$ we are in the most conservative case, because $T_{\mathrm{K}}$ may be higher in position $\mathrm{E}$ and probably in position D. Anyway, the column densities do not vary in more than 10 percent (significantly smaller than other sources of uncertainty) in this temperature range. The first and second columns indicate the position and velocity component. The third column provides the obtained $n\left(\mathrm{H}_{2}\right)$. From fourth to sixth columns, column density of $\mathrm{CO},{ }^{13} \mathrm{CO}$ and $\mathrm{C}^{18} \mathrm{O}$ are provided, respectively.

The results derived for $h v c$ in position $\mathrm{E}$ need to be explained in detail. This velocity component was only detected in the $\mathrm{CO} J=2 \rightarrow 1$ line, and therefore has a minimum $R_{\mathrm{exc}}$ of 6 . 
Such a high value could not be obtained having a $T_{\mathrm{K}}$ of $30 \mathrm{~K}$, even by considering that the emiting region is unresolved by the $12^{\prime \prime}$ beam of the CO $2 \rightarrow 1$ line. Therefore, it is neccesary larger values of $T_{\mathrm{K}}$ and a higher density to match the observed ratio. We estimate that $T_{\mathrm{K}}$ must be larger than $80 \mathrm{~K}$ for this component, as well as the density should be at least $10^{5} \mathrm{~cm}^{-3}$. The undetection of this component in the $\mathrm{CO} J=1 \rightarrow 0$ line strenghtens the idea of a small emiting volume, and a higher excitation temperature is also expected in this position (towards the inner part of the ionized nebula), as discussed in Paper I. Finally, it should be noted that the CO column density for this component has been estimated from the $\mathrm{CO} J=2 \rightarrow 1$ line.

\section{Origin and evolution of the CSM around WR7}

\subsection{Dynamics of the molecular gas around NGC 2359}

The morphology and kinematics of each component, as well as their location with respect to NGC 2359 and the quiescent molecular gas have been discussed in previous sections. Some of the physical conditions have been summarized in Table 2 . In this section, we will put them together in order to draw a picture of the region and its possible evolution.

In Fig. 7, we plot the variation of the gas density and ${ }^{13} \mathrm{CO}$ column density as a function of the projected distance to the W-R star HD 56925, assumed at a distance of $5 \mathrm{kpc}$ (Goudis et al. 1994). The plots correspond just to the $l v c$ and the $i v c$ and not to the $h v c$, because it is only detected at a single position. The stratification of these components are not only evident in the morphology and the kinematics. This progressive variation is also present in the maxium density (roughly $10^{3} \mathrm{~cm}^{-3}$ at $l v c, 10^{4} \mathrm{~cm}^{-3}$ at $i v c$ and at least $10^{5} \mathrm{~cm}^{-3}$ at $h v c$ ) and in the total mass involved (regarding the column densities and the spatial extension of each component). If we take into account the observed low values of $R_{\text {isot }}$, it is also expected a trend in the opacity, decreasing from lvc to hvc. The temperature is expected to increase toward the W-R star, and therefore in the sense $l v c-i v c-h v c$. For the $h v c$, both the density and the temperature must be higher than in the other two components. Although a careful chemical study of the region is needed, we can associate each component to different evolutive episodes previous to the actual W-R status of HD 56925.

In Paper I, we have discovered a quiescent molecular cloud, that was interpreted as the original gas, which was later shocked by the expansion of a wind previous to W-R. The presence of molecular gas close to a W-R star is indeed puzzling, because it is located at a projected distance of only $\sim 8 \mathrm{pc}$, and has been exposed to the very strong radiation and stellar winds during all the evolutive phases of HD 56925. Therefore, the three velocity components reported here are the result of such interaction and represent the disturbed molecular gas linked to some of those phases. In order to associate these features with the $\mathrm{W}-\mathrm{R}$ phase or a previous one, we have made crude estimates of some global dynamical parameters for each component, which are provided in Table 3 . The whole nebula and its surroundings was completely mapped in Paper I, and we considered that the mass obtained there includes both the $l v c$ and the $i v c$. In this work, we roughly estimate that $80 \%$ of that mass

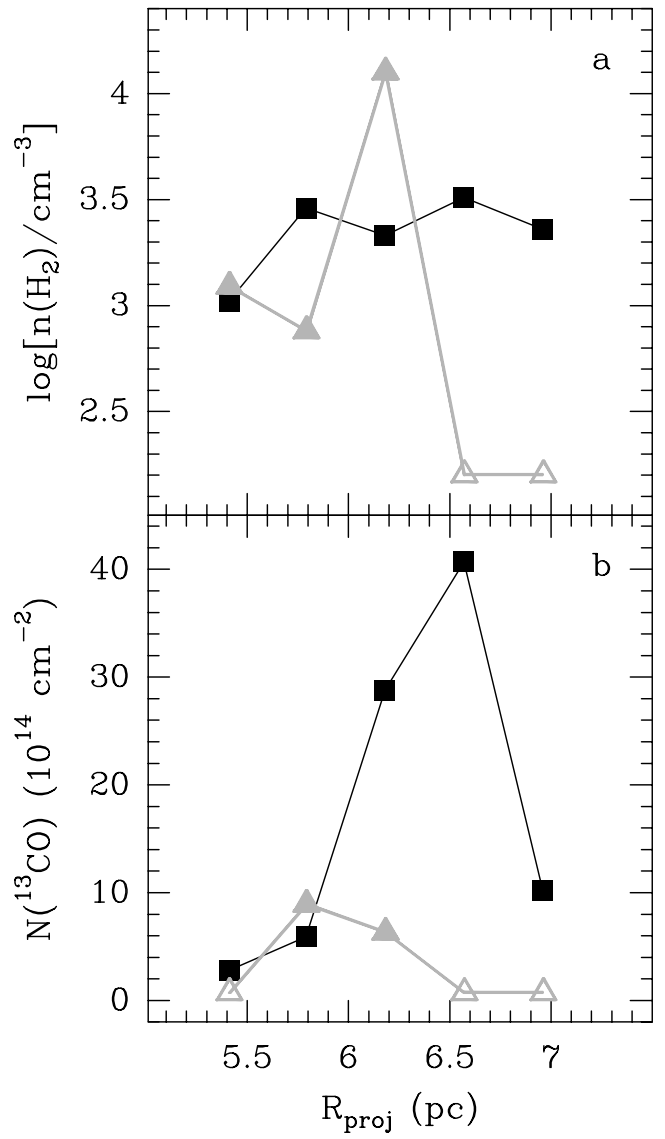

Fig. 7. Variation of some physical parameters for the low (lvc; filled squares) and intermediate (ivc; filled triangles) velocity components, as a function of the distance to HD 56925. a) Distribution of the density (in logarithmic units). b) ${ }^{13} \mathrm{CO}$ column density. Abcissae are projected distance to the W-R star by assuming a distance of $5 \mathrm{kpc}$. Open symbols represent upper limits.

Table 3. Global parameters of the molecular layers in NGC 2359.

\begin{tabular}{lcccccc}
\hline \hline Comp. & $\begin{array}{c}\text { Mass } \\
M_{\odot}\end{array}$ & $\begin{array}{c}V_{\text {exp }} \\
\mathrm{km} \mathrm{s}^{-1}\end{array}$ & $\begin{array}{c}R_{\text {proj }} \\
\mathrm{pc}\end{array}$ & $\begin{array}{c}T_{\text {dyn }} \\
\mathrm{yr}\end{array}$ & $\begin{array}{c}E_{\mathrm{k}} \\
\mathrm{erg}\end{array}$ & $\begin{array}{c}P \\
M_{\odot} \mathrm{km} \mathrm{s}^{-1}\end{array}$ \\
\hline$l v c$ & 130 & 12 & 6.5 & $3.0 \times 10^{5}$ & $2.0 \times 10^{47}$ & $1.7 \times 10^{3}$ \\
$i v c$ & 30 & 17 & 5.8 & $1.9 \times 10^{5}$ & $1.2 \times 10^{47}$ & $6.8 \times 10^{2}$ \\
$h v c$ & 8 & 23 & 5.1 & $1.2 \times 10^{5}$ & $4.8 \times 10^{46}$ & $2.1 \times 10^{2}$ \\
\hline
\end{tabular}

are from the $l v c$, while the other $20 \%$ are from the $i v c$. The mass of the $h v c$ was estimated from the $\mathrm{CO} J=2 \rightarrow 1$ spectra and assuming an angular extension of one beam. The computation of masses and projected distance were done by assuming a distance of $5 \mathrm{kpc}$ (Goudis et al. 1994). The expanding velocities were derived by assuming a rest velocity of $67 \mathrm{~km} \mathrm{~s}^{-1}$ (Paper I). The dynamical time was computed according to Dyson (1989). Finally, Table 3 also includes estimates of kinetic energy and momentum of each component.

The mass is significantly different for each component, decreasing in the sense $l v c-i v c-h v c$, while the other parameters are of the same order of magnitude. All the components have dynamical ages of at most $10^{5} \mathrm{yr}$. This time represents an upper limit of the age of the shock which produced the actual 
Table 4. Evolutive phases of massive stars.

\begin{tabular}{|c|c|c|c|c|c|c|c|}
\hline Phase & $\begin{array}{c}\text { Time } \\
\mathrm{yr}\end{array}$ & $\begin{array}{c}\dot{M} \\
M_{\odot} \mathrm{yr}^{-1}\end{array}$ & $\begin{array}{c}L_{\text {mech }} \\
\text { erg } \\
\end{array}$ & $\begin{array}{c}\dot{P} \\
M_{\odot} \mathrm{km} \mathrm{s}^{-1} \mathrm{yr}^{-1}\end{array}$ & $\begin{array}{c}E_{\mathrm{k}} \\
\mathrm{erg}\end{array}$ & $\begin{array}{c}P \\
M_{\odot} \mathrm{km} \mathrm{s}^{-1} \\
\end{array}$ & $\begin{array}{l}\rho_{\text {wind }} \\
\mathrm{cm}^{-3}\end{array}$ \\
\hline \multicolumn{8}{|c|}{$35 M_{\odot}$, slow wind. Model from García-Segura et al. (1996b) } \\
\hline O-star & $4.5 \times 10^{6}$ & $5.6 \times 10^{-7}$ & $1.1 \times 10^{36}$ & $1.4 \times 10^{-3}$ & $1.6 \times 10^{50}$ & $6.3 \times 10^{3}$ & $2.4 \times 10^{-5}$ \\
\hline RSG & $2.3 \times 10^{5}$ & $8.1 \times 10^{-5}$ & $5.8 \times 10^{33}$ & $1.2 \times 10^{-3}$ & $4.2 \times 10^{46}$ & $2.8 \times 10^{2}$ & $6.8 \times 10^{-1}$ \\
\hline $\mathrm{W}-\mathrm{R}$ & $1.9 \times 10^{5}$ & $2.4 \times 10^{-5}$ & $3.4 \times 10^{37}$ & $5.0 \times 10^{-2}$ & $2.0 \times 10^{50}$ & $9.6 \times 10^{3}$ & $1.2 \times 10^{-3}$ \\
\hline \multicolumn{8}{|c|}{$35 M_{\odot}$, fast wind. Model from García-Segura et al. (1996b) } \\
\hline O-star & $4.5 \times 10^{6}$ & $5.6 \times 10^{-7}$ & $1.1 \times 10^{36}$ & $1.4 \times 10^{-3}$ & $1.6 \times 10^{50}$ & $6.3 \times 10^{3}$ & $2.4 \times 10^{-5}$ \\
\hline RSG & $2.3 \times 10^{5}$ & $8.1 \times 10^{-5}$ & $1.4 \times 10^{35}$ & $6.1 \times 10^{-3}$ & $1.1 \times 10^{48}$ & $1.4 \times 10^{3}$ & $1.4 \times 10^{-1}$ \\
\hline W-R & $1.9 \times 10^{5}$ & $2.4 \times 10^{-5}$ & $3.4 \times 10^{37}$ & $5.0 \times 10^{-2}$ & $2.0 \times 10^{50}$ & $9.6 \times 10^{3}$ & $1.2 \times 10^{-3}$ \\
\hline \multicolumn{8}{|c|}{$60 M_{\odot}$. Model from Langer et al. (1994) } \\
\hline O-star & $1.7 \times 10^{6}$ & $4.7 \times 10^{-6}$ & $1.0 \times 10^{37}$ & $1.2 \times 10^{-2}$ & $5.4 \times 10^{50}$ & $2.1 \times 10^{4}$ & $2.5 \times 10^{-4}$ \\
\hline pre-LBV & $1.5 \times 10^{6}$ & $1.5 \times 10^{-5}$ & $2.7 \times 10^{37}$ & $3.6 \times 10^{-2}$ & $1.4 \times 10^{51}$ & $5.8 \times 10^{4}$ & $9.2 \times 10^{-4}$ \\
\hline LBV & $1.2 \times 10^{4}$ & $6.7 \times 10^{-4}$ & $2.6 \times 10^{37}$ & $2.4 \times 10^{-1}$ & $9.9 \times 10^{48}$ & $2.8 \times 10^{3}$ & $2.4 \times 10^{-1}$ \\
\hline W-R & $6.5 \times 10^{5}$ & $2.4 \times 10^{-5}$ & $3.0 \times 10^{37}$ & $4.8 \times 10^{-2}$ & $6.2 \times 10^{50}$ & $3.1 \times 10^{4}$ & $2.0 \times 10^{-3}$ \\
\hline
\end{tabular}

kinematics. It is one order of magnitude lower than the mainsequence HI shell $\left(2.3 \times 10^{6}\right.$, Paper I $)$, and quite larger than the dynamical age of NGC $2359,1.3 \times 10^{5}$, determined by Treffers \& Chu (1982). However, it should be noted that this dynamical age for the optical nebula is indeed an upper limit, which is reflected by the $1.3-2.2 \times 10^{4} \mathrm{yr}$ determined from Hipparcos proper motion for HD 56925 between the center of the nebula and its actual position. Due to the high symmetry of NGC 2359, we think that a few $10^{4}$ yr represents a reasonable approach of the actual NGC 2359's age. In consequence, the observed $\mathrm{CO}$ features have been produced at an epoch between the end of the main sequence (MS) and the beginning of the W-R phase.

According to the current models, a W-R is the descendant of an O-star of at least $30 M_{\odot}$. Depending on its initial mass, the O-star may evolve to W-R through different evolutive paths, including a red supergiant (RSG) or a luminous blue variable (LBV) phase. Other intermediate stages may account, such as Of or different sub-classes of W-R (WNE, WNL, WC). Although the MS phase is indeed a long-lived one, characterized by a strong stellar wind and an enormous rate of UV-radiation, we could not simply relate the features observed in the molecular gas around NGC 2359 to this phase, untill the other phases were also considered. Moreover, a MS bubble around HD 56925 was found in HI (Paper I), and it has a lineal size of 35-40 pc, closely similar to many other cases in the Galaxy, and in good agreement with the theory.

It is indeed possible that the interaction of the molecular cloud with the MS wind could not be strong enough and the stellar wind could ran through the molecular cloud. This is a reasonable argument owing to the relatively low density of the MS wind. We now consider the other evolutionary phases which may account for the observed features. The more likely mechanism is that the stellar wind has accelerated and heated the quiescent molecular cloud, and hence it is more relevant the instantaneous effect than the accumulated one along the whole phase. Therefore, the phase responsible of accelerating the gas should have a large momentum rate $(\dot{P})$ and perhaps a large mechanical luminosity ( $L_{\text {mech }}$ ). We also should pay attention to the density of the stellar wind, because it is an indicator of how large is, in comparison, the mass injected to the CSM at supersonic velocities.

\subsection{Models of stellar evolution}

Let us consider two different models of stellar evolution, which reproduce two standard evolutive paths: (1) the $35 M_{\odot}$ model of García-Segura et al. (1996b), with the path $\mathrm{O} \rightarrow \mathrm{RSG} \rightarrow \mathrm{W}-\mathrm{R}$; and (2) the $60 M_{\odot}$ model of Langer et al. (1994), with the path $\mathrm{O} \rightarrow$ pre-LBV $\rightarrow \mathrm{LBV} \rightarrow \mathrm{W}-\mathrm{R}$. We should note that some of these phases really includes several other, although no significant changes in the global stellar parameters are noted. So far, the W-R phase in both models can be divided in three sub-stages, such as a H-poor, a $\mathrm{H}$-free and a WC stage. In model (2), the pre-LBV phase includes both the Of and the H-rich WN phase. The evolution of the CSM around both type of stars have been modelled by García-Segura et al. (1996a,b).

The Table 4 provides mean representative values from each phase in each model, such as the time spent, mass loss rate $(\dot{M})$, $L_{\text {mech }}, \dot{P}$, kinetic energy $E_{\mathrm{k}}$ and total momentum $(P)$ provided during the whole phase, and the wind density $\left(\rho_{\text {wind }}\right)$ at $5 \mathrm{pc}$ from the star. The $35 M_{\odot}$ model is split in two cases, having a slow $\left(15 \mathrm{~km} \mathrm{~s}^{-1}\right)$ and a fast $\left(75 \mathrm{~km} \mathrm{~s}^{-1}\right)$ RSG wind.

\subsection{Acummulative effects}

In the three models, both the MS and the W-R stages provide the greatest fraction of the total energy injected to the environs $\left(10^{50-51} \mathrm{erg}\right)$. In the case of the MS stage, however, this is due to an accumulate effect, because the star spents some Myr in MS, one or two orders of magnitude larger than the other phases. In contrast, the W-R stage injects to the medium a similar amount of kinetic energy in some $10^{5} \mathrm{yr}$, because its huge mass-loss rate. On the other hand, the total energy ouput associated to the RSG (fast wind) and LBV phases are two orders of magnitud $\left(10^{48} \mathrm{erg}\right)$ lower than the MS or the W-R stages. 
The amount of kinetic energy associated to the RSG (slow wind) phase is even lower by about 4 orders of magnitude $\left(10^{46} \mathrm{erg}\right)$.

A similar situation occurs when considering the total momentum provided in each phase, although the contrast is lower than in the energy.

\subsection{Instantaneous effects}

When we take into account the instantaneous effects (well described by $L_{\text {mech }}, \dot{P}$ and $\rho_{\text {wind }}$ ), the situation changes dramatically. The intermediate stages RSG and LBV are characterized by an increase of $\dot{M}$ by at least one order of magnitude with respect to their previous phases, and a sudden decrease of the terminal velocity. As a result, $L_{\text {mech }}$ remains almost constant, while both $\dot{P}$ and $\rho_{\text {wind }}$ increases significantly.

During these short-lived phases, the circumstellar gas is filled with a large amount of material $\left(\sim 20 M_{\odot}\right)$ from the stellar interior. This gas is relatively dense even at a few pc from the star and is moving at low (although still supersonic) velocities. The RSG wind (slow wind) is the densest one, but the momentum rate is practically the same as in the MS case; so far, the effect onto the quiescent molecular cloud should not be different from that of the MS stage. The RSG wind (fast wind) is somewhat higher, but not too different from MS wind; a factor of 2 or 3 in these simplified estimates should not be seen as a significant one.

In contrast, the LBV wind is significantly different from the MS wind. The momentum rate is one order of magnitude greater than in any other evolutive stage, and the wind density is three orders of magnitude larger than the previous phases (MS and pre-LBV) and two orders of magnitude larger than the W-R stage.

\subsection{Interpretation}

If the progenitor of the W-R star HD 56925 has had a mass close to $35 M_{\odot}$, the $\mathrm{W}-\mathrm{R}$ wind is the unique source capable of disturbing the molecular cloud, because the RSG wind had not power enough to accelerate the original cloud. So far, the W-R wind had encountered an already stopped dense RSG wind in contact with the molecular cloud. Consequently, the W-R wind has been suddenly decelerated and produced the observed shocked molecular gas.

If, on the other hand, the progenitor of HD 56925 has had a higher mass, it is very probable that the star had developed a LBV phase, which is characterized by a rather dense wind and a high $\dot{P}$. The LBV wind might produce what we are observing in CO. The values provided in Table 4 are mean values for each phase as a whole. This simplification is good enough for all phases but LBV, because the parameters does not significantly change during the whole phase. However, the LBV phase consists of several episodes of enlarged stellar winds (or mass ejections) produced by hydrodynamical instabilities in the stellar structure. It is thought that the mass-loss rate during these episodes reaches values as high as several $10^{-3} M_{\odot} \mathrm{yr}^{-1}$, increasing the mean values of $\dot{M}, \dot{P}, L_{\text {mech }}$ and $\rho_{\text {wind }}$ in Table 4 by a factor $10-20$ during some $10^{3} \mathrm{yr}$. As in the $35 M_{\odot}$ case, the W-R wind may have found the RSG wind and produced the shock observed around the W-R nebula. The presence of several shockfronts (the three $\mathrm{CO}$ components and the ionized layers into the optical nebula) strenghten this idea. If only the W-R stage had produced the observed features, it should have similar episodes of increased mass loss.

The whole optical nebula would be associated to the W-R phase, and its hot material (more or less uniform) would be located inside a multiple-layers wind with an earlier origin. In this case, the bounding of the nebula can be atomic or molecular, depending on the previous presence of a molecular cloud close to the star. The morphology of the molecular gas in NGC 2359, as well as in NGC 6888 and the nebula around WR 134 (Rizzo et al. 2003a) are strongly suggestive in this sense.

In any case, it is clear the need of enlarge the sample, search for possible chemical differences which may confirm our hypothesis, observe and characterize the stellar winds of the wellknown LBVs (P Cyg, $\eta$ Car, HR Car) and construct hydrodynamical models which take into account the different evolutive phases and the presence of a close molecular cloud.

\section{Future perspectives}

The history of a massive star is written by the impacts onto the surrounding gas, particularly in the molecular component. Therefore, we can increase our knowledge about massive stars evolution by observing the consequences in their environments. There are just a few observed cases of interaction between W-R stars and the surrounding molecular gas (Marston et al. 1999; Paper I; Cappa et al. 2001; Rizzo et al. 2003a). However, we think that NGC 2359 becames the best studied case, where a time evolution may be followed and where highdensity gas has been detected. New observational efforts are neccesary in order to increase the sample and confirm some of the results obtained here and predicted by the theory.

The increasing detection of molecular gas around W-R nebulae opens questions which are not completely fulfilled by current models. In the future, new hydrodynamical simulations should address this aspect of the interplay between evolved massive stars and their environs. It is expected to know where and when the molecules can be formed or can survive around these hot massive stars.

On the other hand, the detection and chemical analysis of complex molecules in these ambients, at $\mathrm{mm}$ and sub-mm lines, would tell us about the chemical composition and the excitation conditions of the gas. In this sense, just a few detections have been reported (Marston 2001; Rizzo et al. 2001b, 2003b). This is a key subject, because we can learn not merely about the interplay of the massive star evolution with its surroundings, but also the ambient where a supernova takes place. So far, this field is also connected with the chemical evolution of the Galaxy and its energetic balance. 


\section{Conclusions}

As shown in Paper I, the southeastern border of NGC 2359 contains the most clear sign of interaction of a W-R nebula with the molecular environment. The original molecular cloud was partially accelerated by at least $14 \mathrm{~km} \mathrm{~s}^{-1}$ (from 67 to $53 \mathrm{~km} \mathrm{~s}^{-1}$ ) during one of the early evolutive phases of HD 56925. In this work, we have used the on-the-fly tecnique to obtain detailed maps of the $\mathrm{CO}$ and ${ }^{13} \mathrm{CO}$ emission of this disturbed molecular gas. The angular resolution and sensitivity achieved allowed us to distinguish three velocity components, clearly stratified with respect to the nebula and the quiescent gas.

The first component (called the lvc throughout the paper) is the most extended and the most massive one. The second component, the $i v c$, appears as a thin layer (less than $1 \mathrm{pc}$ thickness) located at the interface between the $l v c$ and the optical nebula. Specially striking is the third component, the $h v c$, which appears as a very compact component, only detected in the CO $J=2 \rightarrow 1$ line towards the inner part of the optical nebula. The $l v c$ has LSR velocities between 52 and $57 \mathrm{~km} \mathrm{~s}^{-1}$ (the nearest to the quiescent gas), the $i v c$ appears at $48-52 \mathrm{~km} \mathrm{~s}^{-1}$, and the $h v c$ emits at velocities between 42 and $48 \mathrm{~km} \mathrm{~s}^{-1}$. So far, the stratification of these components are both morphological and kinematical features.

By observing single positions with higher sensitivity along a strip - more or less radially with respect to WR 7 -, some physical parameters (volume density, column density and opacity) have been inferred. The density increases in the sense $l v c-i v c-h v c$ from $10^{3} \mathrm{~cm}^{-3}$ to at least $10^{5} \mathrm{~cm}^{-3}$, while the column density, total mass and opacity should decrease in the same sense. We have proposed a scenario where the three components are produced during different evolutive episodes of the predecessors of HD 56925. Each component would be the result of an encounter (likely shocks) of the expanding stellar winds (or mass ejections) with the material ejected during the previous phase or even with the ambient gas. This idea qualitatively fits some theoretical results (García-Segura et al. 1996a,b), although some specific aspects should be analyzed in detail. Particularly, the possible presence of molecular gas at a few pc from the massive star should be considered in future theoretical works.

Acknowledgements. This work was partially supported by the Ministerio de Ciencia y Tecnología of Spain (MCyT), by the grant AYA 2003-06473. JM-P acknowledges the financial support from the MCyT grants ESP 2002-01627 and AYA 2002-10113E. J.R.R. acknowledges Dr. M. A. Gómez-Flechoso for very profitable talks about the theoretical models discussed in the paper. The authors are specially grateful with the referee comments, which helped to improve the paper.

\section{References}

Cappa, C. E., Rubio, M., \& Goss, W. M. 2001, AJ, 121, 2664

Castor, J. I. 1970, MNRAS, 149, 111

Chu, Y.-H., Treffers, R. R., \& Kwitter, K. B. 1983, ApJS, 53, 937

Dyson, J. E. 1989, Structure and Dynamics of the Interstellar Medium, IAU Colloq., 120, 137

Esteban, C., Vilchez, J. M., Manchado, A., \& Edmunds, M. G. 1990, A\&A, 227, 515

Flower, D. R., \& Pineau des Forets, G. 1994, MNRAS, 268, 724

García-Segura, G., Langer, N., \& Mac Low, M.-M. 1996a, A\&A, 316, 133

García-Segura, G., Mac Low, M.-M., \& Langer, N. 1996b, A\&A, 305, 229

Goudis, C. D., Christopoulou, P.-E., Meaburn, J., \& Dyson, J. E. 1994, A\&A, 285, 631

Jeffery, D. J. 1995, ApJ, 440, 810

Langer, N., Hamann, W.-R., Lennon, M., et al. 1994, A\&A, 290, 819

Marston, A. P. 2001, ApJ, 563, 875

Marston, A. P., Welzmiller, J., Bransford, M. A., Black, J. H., \& Bergman, P. 1999, ApJ, 518, 769

Mauersberger, R., Guelin, M., Martín-Pintado, J., et al. 1989, A\&AS, 79,217

Rizzo, J. R., Martín-Pintado, J., \& Desmurs, J. F. 2003a, A massive star odyssey, from Main Sequence to Supernova, IAU Symp., 212, 740

Rizzo, J. R., Martín-Pintado, J., \& Desmurs, J. F. 2003b, A massive star odyssey, from Main Sequence to Supernova, IAU Symp., 212, 742

Rizzo, J. R., Martín-Pintado, J., \& Henkel, C. 2001b, ApJ, 553, L181

Rizzo, J. R., Martín-Pintado, J., \& Mangum, J. G. 2001a, A\&A, 366, 146 (Paper I)

St-Louis, N., Doyon, R., Changnon, F., \& Nadeau, D. 1998, AJ, 115, 2475

Schneps, M. H., Haschick, A. D., Wright, E. L., \& Barret, A. H. 1981, ApJ, 243, 184

Treffers, R. R., \& Chu, Y.-H. 1982, ApJ, 254, 569

van der Hucht, K. A. 2001, New Astron. Rev., 45, 135

Wilson, T. L., \& Matteucci, F. 1992, A\&ARv, 4, 1 